\title{
Genomic analyses identify gene candidates for acquired irinotecan resistance in melanoma cells
}

\author{
KAI GAO ${ }^{1}$, WILLIAM W. LOCKWOOD ${ }^{2}$, JUN LI $^{1}$, WAN LAM ${ }^{2}$ and GANG LI ${ }^{1}$ \\ ${ }^{1}$ Department of Dermatology and Skin Science, Jack Bell Research Centre, Vancouver Coastal Health Research Institute, \\ University of British Columbia; ${ }^{2}$ Department of Cancer Genetics and Developmental Biology, \\ British Columbia Cancer Research Centre, Vancouver, BC, Canada
}

Received February 8, 2008; Accepted March 28, 2008

\begin{abstract}
Efficacy of camptothecins (CPTs) such as irinotecan has been recognized in chemotherapy of cancers including melanoma. However, the majority of responding patients will gradually acquire drug resistance. Little is known of the genes responsible for the acquired CPTresistance in cancer. To gain global insight into acquired CPT-resistance, we established irinotecan-resistant clones derived from melanoma cells and compared their whole genomes by high resolution array-CGH. A novel gain at 14q23.2-31.1 was revealed by alignment of whole genome profiles of parental cell line and irinotecan-resistant clones. Further analysis of this amplicon indicates that it encompassed genes involved in DNA repair $(R A D 51 L$, $M L H 3)$, reactive oxygen species $(G P X 2, C S T Z 1, N G B$, $R D H 11, Z A D H 1)$, and transportome (ABCD4, ATP6V1D, $S L C 10 A 6)$. Moreover, losses were also detected at the loci of topoisomerases (TOP1, SPO1 1, TOP3B) as well as at the loci of genes guarding chromosomal stability (TP53, ZW10, $H 2 A F X, C H K 1, C C D N 1, M C M 5, C E N P B, D N M T 3 B)$, which would facilitate the development of drug resistance. Furthermore, quantitative real-time PCR demonstrated that mRNA changes of selected novel genes (CENPB, H2AFX, $M C M 5, Z A D H 1$ and $N G B)$ in irinotecan-resistant clones vs. parental clone were in agreement with array-CGH results. Taken together, our data suggest that genes involved in genome stability may greatly contribute to the development of CPTs-resistance. In addition, genes located at 14q23.331.1 would be promising targets to overcome acquired CPT-resistance in melanoma.
\end{abstract}

Correspondence to: Dr Gang Li, Department of Dermatology and Skin Science, Jack Bell Research Centre, 2660 Oak Street, Vancouver, BC V6H 3Z6, Canada

E-mail: gangli@interchange.ubc.ca

Key words: irinotecan, melanoma, array CGH, topoisomerase, drug resistance

\section{Introduction}

Cutaneous malignant melanoma is the most aggressive form of skin cancer and its incidence has been rising rapidly. In 2007 , the number of new melanoma cases was estimated to be 59,940 and it was predicted to have 8,110 deaths due to melanoma in USA (1). If melanoma is not diagnosed at early stage, the survival rate decreases rapidly (2).

Camptothecins (CPTs), such as irinotecin and SN-38, have been widely used for treating cancer patients and their efficacy has been recognized for years. Recent studies indicated that new generation of CPT, karenitecin (BNP1350), has a promising response rate in melanoma patients (3). However, responded patients gradually develop drug resistance within a few months. Therefore, it is essential to identify the genes responsible for the development of CPT-resistance to improve their efficacy in cancer treatment. Previous studies indicated that topoisomerase I (TOP1) is the direct target of CPTs. Altered expression or mutation of TOP1 in cancer cells is associated with CPT-resistance (4). In addition, studies have revealed that CPTs inhibit both DNA and RNA synthesis, arrest cells in G2 phase of cell cycle, induce chromosomal aberrations and elevate p53 levels (5-8). However, little is known for the mechanisms of acquired CPT-resistance and the relationship between intrinsic and acquired CPT resistance in cancer cells. In the present study, we obtained a global view of acquired irinotecan-resistance in melanoma cells using high resolution array-CGH technology to analyze chromosomal alterations of irinotecin-resistant cell lines vs. their parental cell line. We found a prominent gain at $14 \mathrm{q}$ and losses at loci of topoisomerases in irinotecan-resistant cell lines compared to the parental cells. In addition, changes were found in loci of a number of genes which influence chromosomal stability as well as regulation of DNA repair and reactive oxygen species.

\section{Materials and methods}

Cell lines and culture conditions. MMRU melanoma cell line (a kind gift from Dr H.R. Byers, Boston University), which contains the wild-type $p 53$ gene $(9,10)$ was maintained in Dulbecco's modified Eagle's medium supplemented with $10 \%$ fetal bovine serum (Invitrogen, Burlington, ON, Canada). To 
Table I. Primer pairs used in qRT-PCR.

\begin{tabular}{|c|c|c|}
\hline Target gene & & Primer sequence \\
\hline \multirow[t]{2}{*}{$C E N P-B$} & Forward & 5'-GCCACATCCTCCACTTGGAA-3' \\
\hline & Reverse & 5'-GTGACTCAGCTTTGATGTCCAAGA-3' \\
\hline \multirow[t]{2}{*}{$H 2 A F X$} & Forward & 5'-CTTGGTAACAGGCACATCTTCCT-3' \\
\hline & Reverse & 5'-CCACATAGCCAGCCGTGAA-3' \\
\hline \multirow[t]{2}{*}{ MCM5 } & Forward & 5'-GACCCTTCGTCCCGGAATT-3' \\
\hline & Reverse & 5'-CATCTTCTCGCATCTTGTCAAACT-3' \\
\hline \multirow[t]{2}{*}{$\mathrm{ZADH1}$} & Forward & 5'-GCCTGGCATTCTACAGCTGAGT-3' \\
\hline & Reverse & 5'-CTGGAATGCAGCTCCCATGT-3' \\
\hline \multirow[t]{2}{*}{$N G B$} & Forward & 5'-GCTGCCCCTCTTCCAGTACA-3' \\
\hline & Reverse & 5'-CACGAGCATCACCTTCCTGAT-3' \\
\hline \multirow[t]{2}{*}{$A C T I N$} & Forward & 5'-GCTCTTTTCCAGCCTTCCTT-3' \\
\hline & Reverse & 5'-CGGATGTCAACGTCACACTT-3' \\
\hline
\end{tabular}

select irinotecan-resistance subclones, MMRU cells were exposed to increasing doses of irinotecan (starting at $0.01 \mu \mathrm{M}$ for 12 months and then cultured in complete medium containing $0.02 \mu \mathrm{M}$ irinotecan). All cells were maintained in $5 \% \mathrm{CO}_{2}$ atmosphere at $37^{\circ} \mathrm{C}$.

Sulforhodamine B cell survival assay. Cells were seeded into 24-well plates at a concentration of $2.5 \times 10^{4}$ cells per well. After $24 \mathrm{~h}$, cells were treated with different concentrations of irinotecan for $48 \mathrm{~h}$. Relative toxicity was determined by the sulphorhodamine (SRB) assay (11).

Clonogenic assay. Cells were seeded at a density of 500 cells/ well in 6-well plates and allowed to attach for $24 \mathrm{~h}$. Then cells were treated with various doses of irinotecan. After 24-h exposure, the cells were fed with drug-free medium and allowed to grow for approximately 10-15 days. Cells were then fixed with $3.7 \%$ formalin and stained with $2 \%$ crystal violet, and colonies that contain $>50$ cells were scored.

DNA extraction and tiling path array comparative genomic hybridization. DNA was extracted using standard proteinase K/RNase treatment and phenol-chloroform extraction. The construction of SMRT array and performance of array hybridization were previously described (12). Briefly, $400 \mathrm{ng}$ of sample and reference genomic DNA (Novagen, Mississauga, ON, Canada) was separately labeled by random priming in the presence of cyanine-3 and cyanine-5 dCTP (PerkinElmer, Waltham, MA), respectively. Labeled sample and reference DNA were combined and purified using ProbeQuant Sephadex G-50 Columns (Amersham, Baie d'Urfe, PQ, Canada) followed by addition of $100 \mu \mathrm{g}$ Cot-1 DNA (Invitrogen) and precipitation with $3 \mathrm{M}$ sodium acetate and $100 \%$ ethanol. The DNA pellet was resuspended in $45 \mu 1$ of hybridization solution containing $80 \%$ DIG Easy hybridization buffer (Roche, Laval, PQ, Canada), $100 \mu \mathrm{g}$ sheared herring sperm DNA (Sigma-Aldrich, St. Louis, MO) and $50 \mu$ g yeast tRNA (Calbiochem, San Diego, CA), and then denatured at $85^{\circ} \mathrm{C}$ for $10 \mathrm{~min}$. Repetitive sequences were blocked at $45^{\circ} \mathrm{C}$ for $1 \mathrm{~h}$ prior to hybridization and added to array slides and placed in a prewarmed hybridization buffer (Telechem, Sunnyvale, CA). After hybridization for $40 \mathrm{~h}$ at $45^{\circ} \mathrm{C}$, arrays were washed five times for $5 \mathrm{~min}$ each in $0.1 \mathrm{X} \mathrm{SSC} / 0.1 \% \mathrm{SDS}$ at room temperature in the dark with agitation followed by five rinses in $1.0 \mathrm{X}$ SSC and dried using an oil-free air stream.

Imaging and data analysis. Images of the hybridized array were captured through cyanine- 3 and cyanine- 5 channels using a charge-coupled device (CCD) camera system (Applied Precision, Issaquah, WA). Images were analyzed using Soft-WoRx Tracker Spot Analysis software (Applied Precision). Systematic array biases were removed using a stepwise normalization procedure. Custom software called SeeGH was used to combine replicates and visualize all data as $\log _{2}$ ratio plots in SeeGH karyograms. All spots with standard deviations above 0.1 were removed from the analysis (12).

RNA extraction and quantitative real-time PCR. Cells were lysed in TRIzol (Invitrogen, Carlsbad, CA) and total RNA was isolated according to the manufacturer's protocol. For validation of array results, cDNA was obtained using a combination of oligo-dT, dNTP mix (10 nM each) and Superscript II (Invitrogen). PCR primers for each gene (CENPB, H2AFX, $M C M 5, Z A D H 1$ and $N G B$ ) (Table I) were designed using Primer Express 3.0 software (Applied Biosystems, Foster City, CA), with a melting temperature at $58-60^{\circ} \mathrm{C}$. PCR of each primer was carried out in triplicate in $25 \mu \mathrm{l}$ using SYBR- Green Master Mix (Applied Biosystems) for $15 \mathrm{~min}$ at $95^{\circ} \mathrm{C}$ for initial denaturing, followed by 40 cycles of $95^{\circ} \mathrm{C}$ for $30 \mathrm{sec}$ and $60^{\circ} \mathrm{C}$ for $30 \mathrm{sec}$ in the ABI $7900 \mathrm{HT}$ fast realtime PCR System. 
A

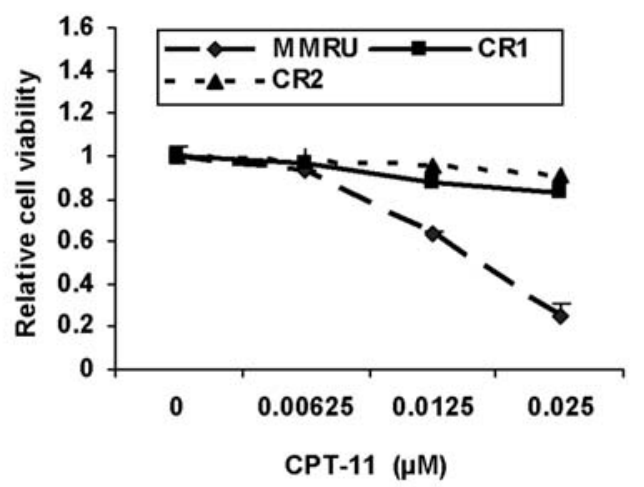

B

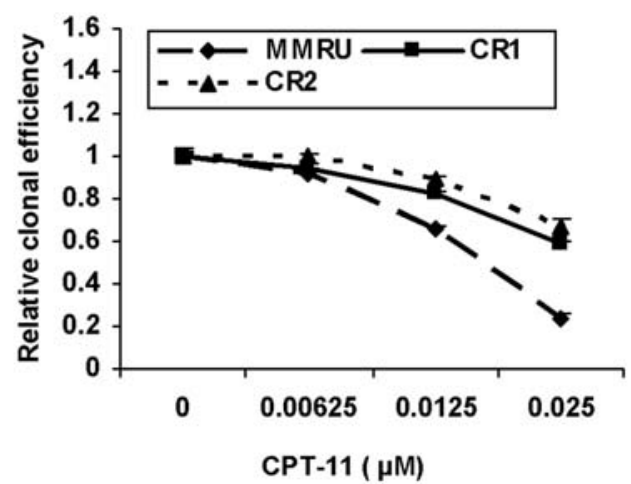

Figure 1. CR1 and CR2 cell lines with CPT-11 resistance derived from their parental MMRU cell line. (A) Acute cytotoxicity of irinotecan in MMRU, CR1 and CR2 cell lines. (B) Clonogenic survival of MMRU, CR1 and CR2 cell lines after irinotecan treatment. The curves show the means and standard deviations of at least three independent experiments.
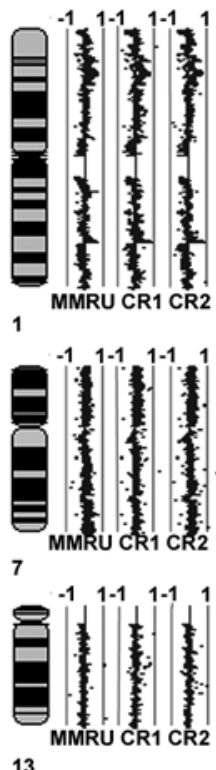

13

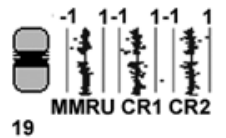

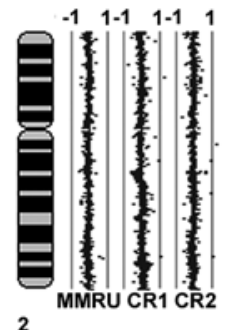

2
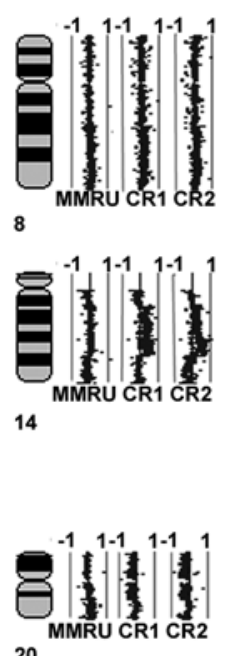
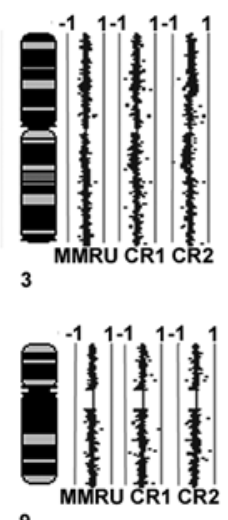

9
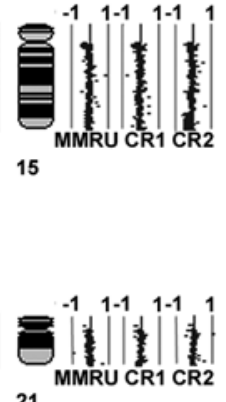
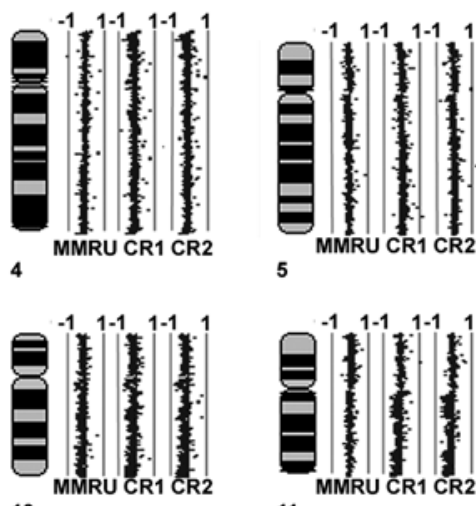

10

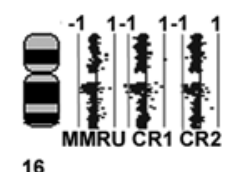

16

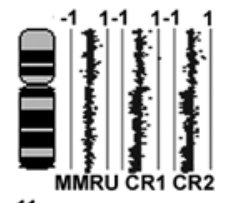

11
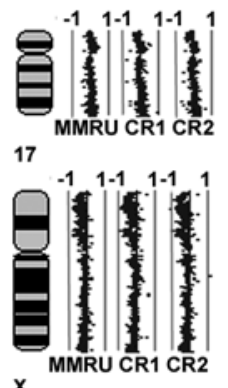
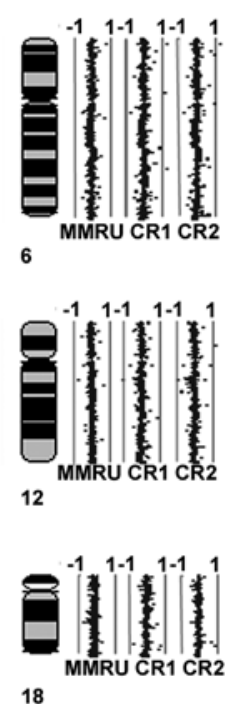

18

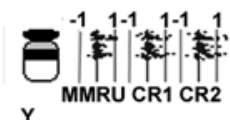

Figure 2. Whole genome array CGH profiles of MMRU and its derived cell lines CR1 and CR2.

Results

Generation of acquired resistance to irinotecan in human melanoma MMRU cell line. MMRU melanoma cell line was exposed to increasing concentration of irinotecan and the derived cell lines CR1 and CR2 were generated over 12-month exposure. The morphology of CR1 and CR2 is similar to their parental MMRU cell line. However, CR1 and CR2 cells are relatively larger than the parental MMRU cells. The higher survival rates of CR1 and CR2 after exposing to different doses of irinotecan indicated their resistance compared to the parental MMRU cell line (Fig. 1A). Colonogenic assay further confirmed that CR1 and CR2 cell lines are less sensitive to irinotecan than the parental cell line (Fig. 1B).

Whole genomic tiling array CGH profiles of parental MMRU cell line and its derived irinotecan-resistant cell lines $C R 1$ and $C R 2$. We characterized the segmental DNA copy number status of MMRU, CR1 and CR2 using tiling-path array comparative genomic hybridization (CGH) technology, which evaluates genetic alteration at $\sim 26,000$ loci completely covering the sequenced whole human genome. Signal intensity ratio for each clone was calculated and normalized in SeeGH visualization software. Fig. 2 displays the SeeGH karyogram 


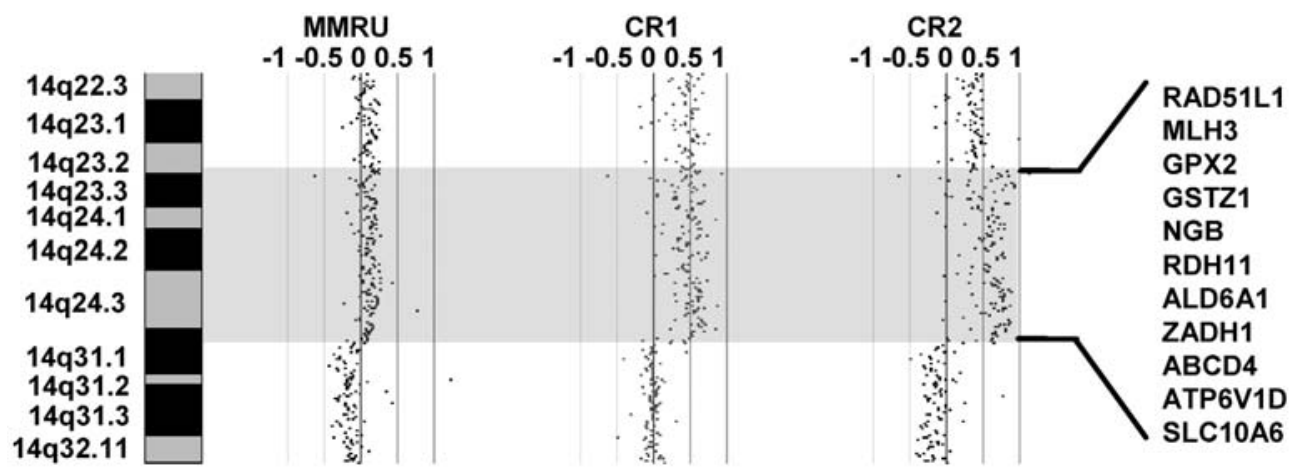

Figure 3. Loci gain of genes involved in DNA repair, reactive oxygen species and transportome (transporters and channels) at 14q23.2-31.1 in CR1 and CR2 cell lines vs. the parental MMRU cell line.

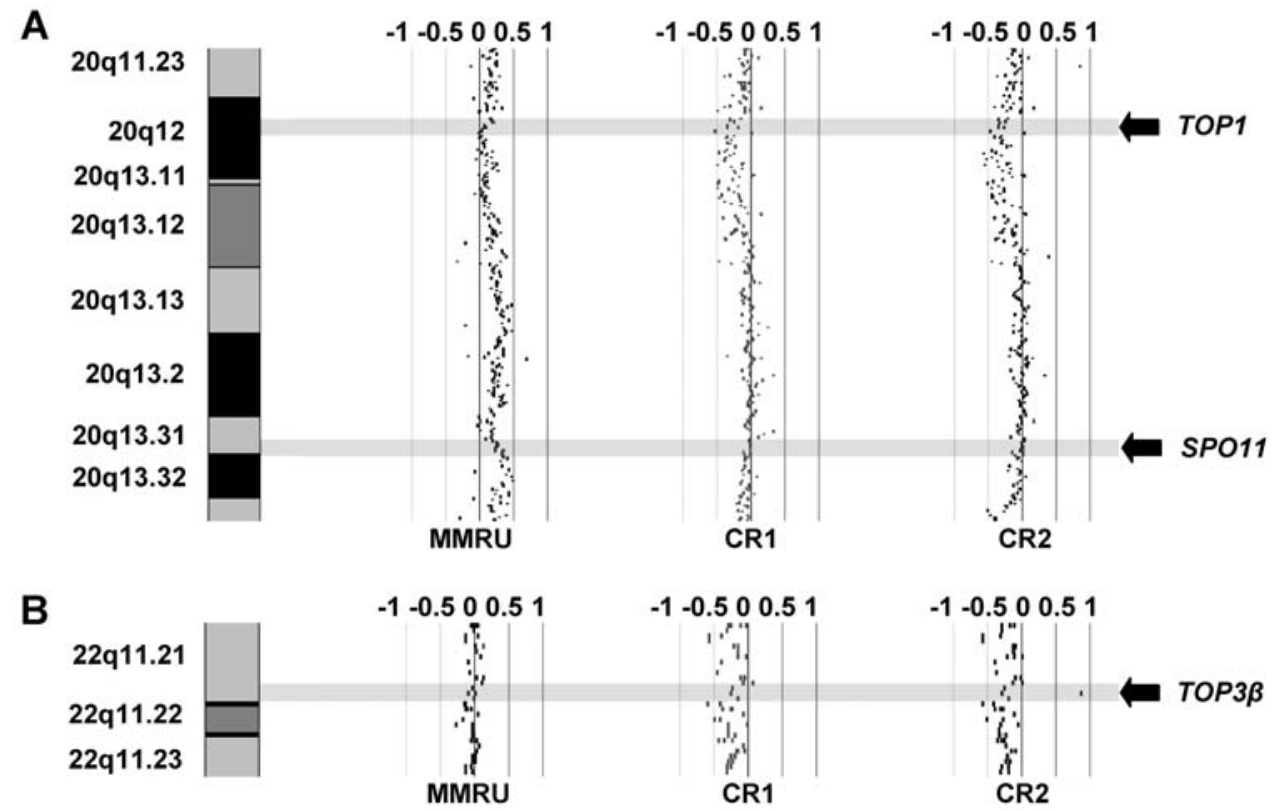

Figure 4. Loci loss of topoisomerases (TOP1, SPO11 and TOP3ß) in CR1 and CR2 cell lines vs. the parental MMRU cell line.

of MMRU, CR1 and CR2. Some chromosomes such as chromosome 1, 5, 7 are almost identical among the profiles of MMRU, CR1 and CR2, confirming that CR1 and CR2 clones are derived from their parental cell line MMRU. On the other hand, large alterations such as the gain of $14 q$ and loss of $20 \mathrm{q}$ are evident.

Genomic copy number gain at 14q23.2-31.1 encompassing genes in DNA repair, reactive oxygen species (ROS), and transportome. Since there is an obvious amplicon at $14 \mathrm{q} 23.2-$ 31.1 , we further analyzed which loci of genes may increase their copy numbers. We detected gain at loci of following genes: RAD51L1 and $M L H 3$, which promote DNA repair; GPX2, GSTZ1, NGB, RDH11, ALDH6A1 and ZADH1, which regulates the generation of ROS; $A B C D 4, A T P 6 V 1 D$, SLC10A6, which are transportome genes (Fig. 3).

Genetic copy number losses at loci of topoisomerases. We also investigated whether the loci of topoisomerases are changed in karyogram of CPT-resistant cell line CR1 and
CR2 vs. the parental cell line MMRU, since TOP1 is the direct target of CPTs. We screened four type I topoisomerases (TOP1, TOP1MT, TOP $3 \alpha$ and TOP $3 \beta$ ), three type II topoisomerases (TOP $2 \alpha, T O P 2 \beta$ and $S P O 11)$ as well as topoisomerase II binding protein. We found that the loci of TOP1, TOP $3 \beta$ and SPO11 show great loss (Fig. 4) while others display no or marginal difference between the profiles of CR1/CR2 and that of MMRU cell line.

Genetic copy number losses at loci of genes controlling chromosome stability. Considering obvious alteration in genomic profiles of irinotecan-resistant clones CR1 and CR2 compared to parental MMRU cell line, we, therefore, investigated if there are changes at loci of genes guarding chromosomal stability. We first detected a loss at the locus of $p 53$, the guardian of the genome (Fig. 5A). In addition, we screened the genes regulating cell cycle, mitosis or turnover of histones, which are all reported to be responsible for chromosomal stability. We found that detectable losses at loci of following genes: $C H K 1$ and $C C D N 1$ (Fig. 5B and C), 


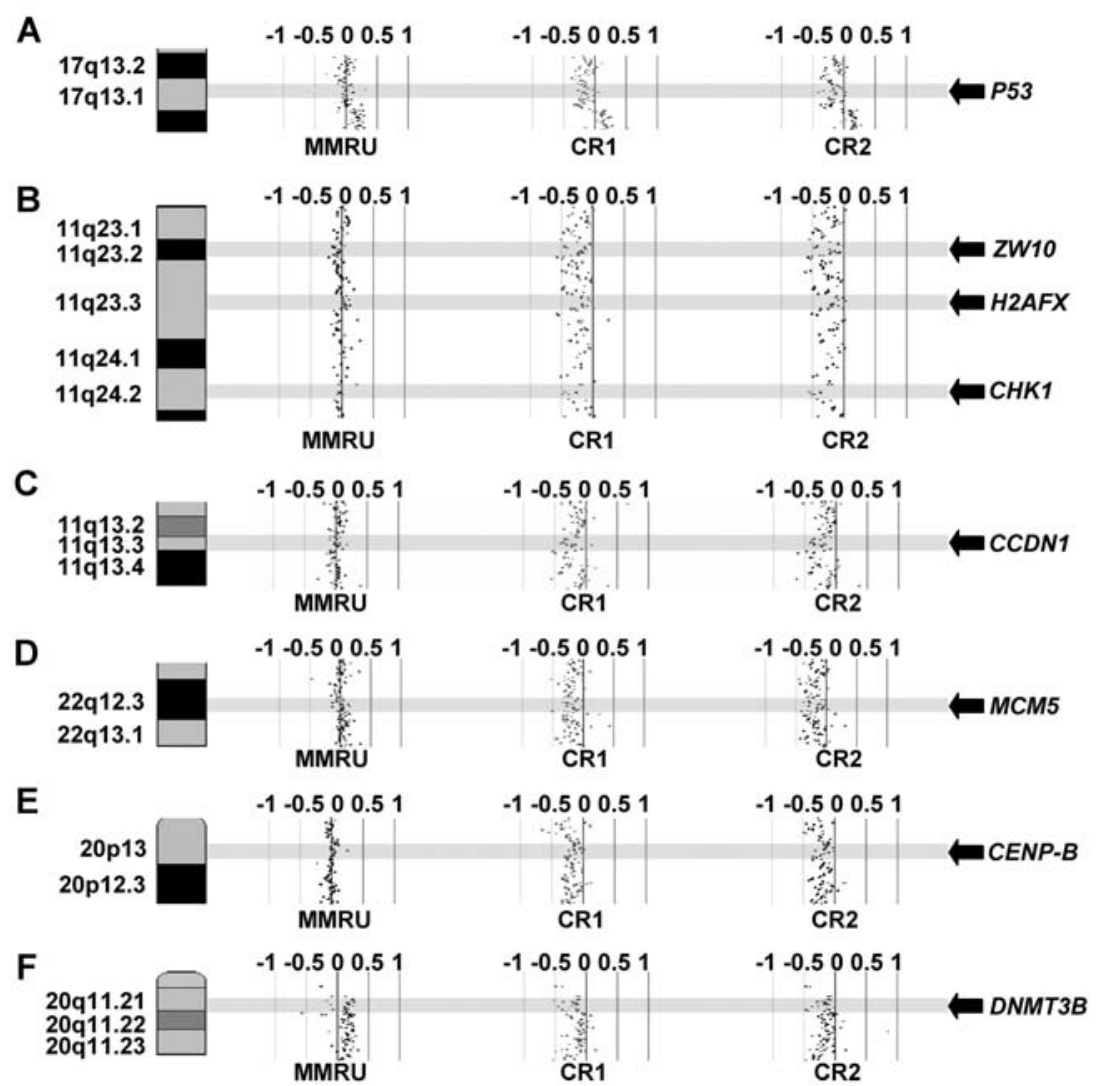

Figure 5. Loci loss of genes guarding chromosomal stability in CR1 and CR2 cell lines vs. the parental MMRU cell line.

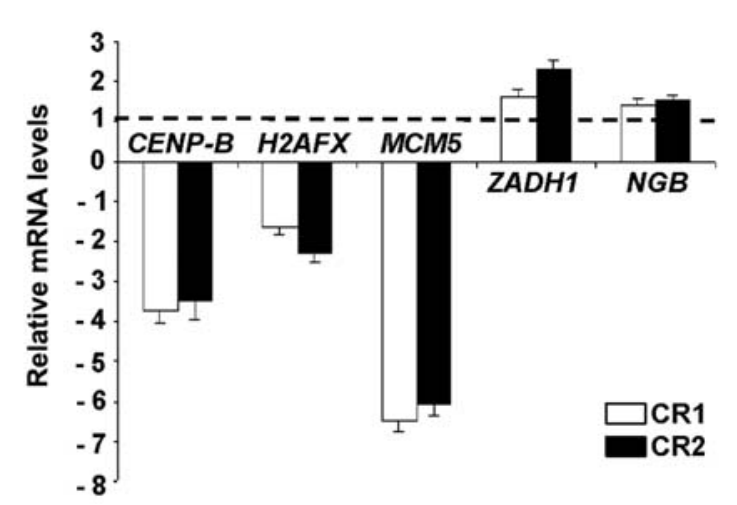

Figure 6. Validation of selected genes in CR1 and CR2 cell lines vs. the parental MMRU cell line. The horizontal dashed line indicates the levels of expression of genes in MMRU cell line, which is arbitrarily set to 1 . The two columns represent gene expression levels in CR1 and CR2 cell lines, relative to expression levels in their parental MMRU cell line. Columns, means of triplicate experiments; bars, SD.

important mediators in cell cycle regulation; $Z W 10, M C M 5$, CENP-B (Fig. 5B, D and E), mitosis-related genes; and $H 2 A F X$ and DNMT3B (Fig. 5B and F), which influence the modification of histones and methylation status of the genome, respectively $(13,14)$.

Expression validation of selected genes by quantitative $R T$ $P C R$. To examine the reliability of changes of loci identified by array-CGH, we selected 5 novel genes which are involved in CPT-resistance: CENPB, H2AFX, MCM5, ZADH1 and
$N G B$ for real-time PCR analysis. The trend of expression changes of these genes are in agreement with array $\mathrm{CGH}$ data (Fig. 6).

\section{Discussion}

Although TOP1 is a well-known direct target of CPTs and its mutation is associated with CPT-resistance, studies have indicated that other genes, which regulate cell cycle, apoptosis, ROS and transporters, all play a role in CPT-resistance $(6,9,15)$. Since majority of studies focused on the function of a single gene, we sought to gain a global view and identify specific gene candidates involved in the development of acquired CPT-resistance. Using high resolution array-CGH, we aligned the genome profile of parental cancer cell line and its derived CPT-resistant clones. We found a novel amplicon at 14q23.3-31.1 as well as a number of genes involved in genome instability.

Using UCSF genome browser, we screened genes located at 14q23.3-31.1. We found this amplicon concentrates genes regulating reactive oxygen species (ROS) including $G P X 2$, GSTZ1, NGB, RDH11, ALDH6A1 and ZADH1 (Fig. 3). Cancer cells with high level of ROS show natural resistance to anticancer drugs (16). However, ROS burst or ROS stress level beyond a 'threshold', which was induced by high dose of anticancer drug or inhibitors of redox enzymes such as 2-methoxyestradiol, an inhibitor of superoxide dismutase (SOD), would lead to apoptosis of cancer cells $(17,18)$. Therefore, it could be assumed that NGB, GPX2, ALDH6A1 and GSTZ1 genes, which were identified in acquired 
irinotecan-resistant clones, would function together as radical scavengers to eliminate ROS induced by chronic exposure of irinotecan and contribute to chemoresistance. In addition, we identified gains at loci of two genes involved in DNA repair, RAD51L1 and $M L H 3$, one genes of SLC transporter, SLC10A6; one gene of ion pump, ATP6V1D. Since enhanced DNA repair and transportome activity have been reported to be related to natural drug resistance $(6,15)$, it implies that acquired irinotecan-resistant clones may also employ similar mechanisms for drug resistance and these related genes can be considered as specific targets in the future study.

As expected, the loss at loci of TOPI gene is detected in the profiles of irinotecan-resistant clones CR1 and CR2 compared to the parental cell line MMRU (Fig. 4A). In addition, we observed losses at loci of TOP $3 \beta$, a type I topoisomerase and SPO11, a type II topoisomerase (Fig. 4). There were no detectable changes at loci of other topoisomerases (data not show). These data imply that resistant clones could gain multiple drug resistance, which not only bypasses TOP I inhibitor such as irinotecan, but also overcomes TOP II inhibitors such as doxorubicin and etoposide in multidrug resistance.

Prominent alterations of chromosomes in CR1 and CR2 cell lines vs. the parental cell line prompted us to investigate loci changes at genes guarding chromosomal stability which facilitate cancer cells to adapt to new microenvironment and enhance their chemoresistance $(19,20)$. We detected genetic copy number losses at loci of p53 and CHK1 in CR 1 and CR2 cell lines (Fig. 5A and B). Wild-type $p 53$ and CHK1 are activated by acute exposure to CPTs, and thus induce cell cycle arrest at $\mathrm{S}$ and $\mathrm{G} 2 / \mathrm{M}$ phases, which allows cells to repair CPT-induced DNA damage before mitosis (21). However, during the development of acquired CPT-resistance, p53 and CHK1 are lost, so the resistant clones can bypass irinotecaninduced cell cycle arrest and continue to divide. Although CHK1 inhibitors have been shown to enhance the cytotoxicity of CPT-11 and etoposide in some cancer cells in transient manner $(22,23)$, our data suggest that these inhibitors may not work effectively in patients with acquired CPT-resistance. Additionally, we observed a loss at loci of the MCM5 gene (Fig. 5D) and its decreased expression in CR1 and CR2 clones (Fig. 6). A recent report also found that MCM5 expression was inhibited when a p53-mutant breast cancer cell line was treated with CPT-11 and CHK1 inhibitor simultaneously (23) further confirming that compromised checkpoints in acquired irinotecan-resistant clones facilitates loss of chromosomal stability and their plasticity to CPT stress.

Our data also indicate losses of $H 2 A F X$ and DNMT3B genes in acquired irinotecan-resistant clones (Fig. 5B and F). $H 2 A F X(H 2 A X)$ is a member of the histone H2A family, basic nuclear proteins responsible for the nucleosome structure in eukaryotes. $H 2 A F X$ was quickly phosphorylated and located at sites of DNA damage (24). Cells from $H 2 A F X$ knock-out mice showed chromosomal abnormality after DNA damage (25). DNMT3B is a DNA methyltransferase, which is essential for de novo methylation and embryonic development (26). Mice with point mutation of DNMT3B gene exhibits ICF (Immunodeficiency, Centromeric instability and Facial anomalies) syndrome (27), implying that DNMT3B is vital in cell cycle and mice development. Therefore, we postulated that loci changes of H2AFX and DNMT3B would directly interfere with genome structure and enhance chromosomal instability.

Taken together, our data suggest that alterations of the genes involved in genome stability may contribute to the development of CPTs-resistance. In addition, genes located at 14q23.3-31.1 are promising targets to overcome acquired CPT-resistance in melanoma.

\section{Acknowledgements}

We thank Dr H.R. Byers for providing the MMRU cell line, Dr William Jia for advice on establishment of drugresistant cell lines and Ariane Williams for technical support on array-CGH. This work was supported by the Canadian Dermatology Foundation.

\section{References}

1. Jemal A, Siegel R, Ward E, et al: Cancer statistics, 2007. CA Cancer J Clin 57: 43-66, 2007.

2. Eigentler TK, Caroli UM, Radny $P$ and Garbe C: Palliative therapy of disseminated malignant melanoma: a systematic review of 41 randomised clinical trials. Lancet Oncol 4: 748-759, 2003.

3. Daud A, Valkov N, Centeno B, et al: Phase II trial of karenitecin in patients with malignant melanoma: clinical and translational study. Clin Cancer Res 11: 3009-3016, 2005.

4. $\mathrm{Xu} \mathrm{Y}$ and Villalona-Calero MA: Irinotecan: mechanisms of tumor resistance and novel strategies for modulating its activity. Ann Oncol 13: 1841-1851, 2002.

5. Hsiang YH, Hertzberg R, Hecht S and Liu LF: Camptothecin induces protein-linked DNA breaks via mammalian DNA topoisomerase I. J Biol Chem 260: 14873-14878, 1985.

6. Tsao YP, D'Arpa P and Liu LF: The involvement of active DNA synthesis in camptothecin-induced G2 arrest: altered regulation of p34cdc2/cyclin B. Cancer Res 52: 1823-1829, 1992.

7. Huang CC, Han CS, Yue XF, et al: Cytotoxicity and sister chromatid exchanges induced in vitro by six anticancer drugs developed in the People's Republic of China. J Natl Cancer Inst 71: 841-847, 1983.

8. Nelson WG and Kastan MB: DNA strand breaks: the DNA template alterations that trigger p53-dependent DNA damage response pathways. Mol Cell Biol 14: 1815-1823, 1994.

9. Li G, Bush JA and Ho VC: p53-dependent apoptosis in melanoma cells after treatment with camptothecin. J Invest Dermatol 114: 514-519, 2000

10. Gao K, Dai DL, Martinka M and Li G: Prognostic significance of nuclear factor- $\{$ kappa 3 B 105/p50 in human melanoma and its role in cell migration. Cancer Res 66: 8382-8388, 2006.

11. Li G, Tang L, Zhou X, Tron V and Ho V: Chemotherapy-induced apoptosis in melanoma cells is p53 dependent. Melanoma Res 8: 17-23, 1998.

12. Lockwood WW, Coe BP, Williams AC, MacAulay C and Lam WL: Whole genome tiling path array $\mathrm{CGH}$ analysis of segmental copy number alterations in cervical cancer cell lines. Int J Cancer 120: 436-443, 2007.

13. Costelloe T, Fitzgerald J, Murphy NJ, Flaus A and Lowndes NF: Chromatin modulation and the DNA damage response. Exp Cell Res 312: 2677-2686, 2006.

14. Luczak MW and Jagodzinski PP: The role of DNA methylation in cancer development. Folia Histochem Cytobiol 44: 143-154, 2006.

15. Huang Y, Anderle P, Bussey KJ, et al: Membrane transporters and channels: role of the transportome in cancer chemosensitivity and chemoresistance. Cancer Res 64: 4294-4301, 2004.

16. Zhou Y, Hileman EO, Plunkett W, Keating MJ and Huang P: Free radical stress in chronic lymphocytic leukemia cells and its role in cellular sensitivity to ROS-generating anticancer agents. Blood 101: 4098-4104, 2003.

17. Kong Q, Beel JA and Lillehei KO: A threshold concept for cancer therapy. Med Hypotheses 55: 29-35, 2000.

18. Huang P, Feng L, Oldham EA, Keating MJ and Plunkett W: Superoxide dismutase as a target for the selective killing of cancer cells. Nature 407: 390-395, 2000. 
19. Snijders AM, Fridlyand J, Mans DA, et al: Shaping of tumor and drug-resistant genomes by instability and selection. Oncogene 22: 4370-4379, 2003

20. Swanton C, Tomlinson I and Downward J: Chromosomal instability, colorectal cancer and taxane resistance. Cell Cycle 5: 818-823, 2006.

21. Deptala A, Li X, Bedner E, et al: Differences in induction of p53, p21WAF1 and apoptosis in relation to cell cycle phase of MCF-7 cells treated with camptothecin. Int J Oncol 15: 861-871, 1999.

22. Maude SL and Enders GH: Cdk inhibition in human cells compromises chk1 function and activates a DNA damage response. Cancer Res 65: 780-786, 2005.

23. Fanton CP, Rowe MW, Moler EJ, et al: Development of a screening assay for surrogate markers of CHK1 inhibitor-induced cell cycle release. J Biomol Screen 11: 792-806, 2006.
24. Furuta T, Takemura H, Liao ZY, et al: Phosphorylation of histone $\mathrm{H} 2 \mathrm{AX}$ and activation of Mre11, Rad50 and Nbs1 in response to replication-dependent DNA double-strand breaks induced by mammalian DNA topoisomerase I cleavage complexes. J Biol Chem 278: 20303-20312, 2003.

25. Bogliolo M, Lyakhovich A, Callen E, et al: Histone H2AX and Fanconi anemia FANCD2 function in the same pathway to maintain chromosome stability. EMBO J 26: 1340-1351, 2007.

26. Okano M, Bell DW, Haber DA and Li E: DNA methyltransferases Dnmt3a and Dnmt3b are essential for de novo methylation and mammalian development. Cell 99: 247-257, 1999.

27. Ueda Y, Okano M, Williams C, et al: Roles for Dnmt3b in mammalian development: a mouse model for the ICF syndrome. Development 133: 1183-1192, 2006. 